\title{
Development of individual medical implants with specific mechanical properties manufactured by Laser Powder Bed Fusion
}

\author{
Johannes Willkomm ${ }^{1}$, Lucas Jauer ${ }^{2}$, Stephan Ziegler ${ }^{1}$, Johannes Henrich \\ Schleifenbaum ${ }^{1,2}$ \\ ${ }^{1}$ RWTH Aachen University - Digital Additive Production \\ ${ }^{2}$ Fraunhofer Institute for Laser Technology ILT \\ johannes.willkomm@dap.rwth-aachen.de, \\ lucas.jauer@ilt.fraunhofer.de, stephan.ziegler@dap.rwth-aachen.de, \\ johannes.henrich.schleifenbaumedap.rwth-aachen. de
}

\begin{abstract}
Laser Powder Bed Fusion (LPBF) is an additive manufacturing process, which enables the generation of complex geometries such as lattice structures, using metallic powder. Lattice structures are being used increasingly in medical technology to adapt the stiffness of individualized implants which can lead to faster bone healing. Lattice structures are also used to adjust the contact surface between the bone and implant to adapt the osseointegrative behavior. The goal of this work is to create lattice structures with local adaption of the stiffness (modulus of elasticity) for individual vertebral body replacement implants and their automated design based on patient data.

To form the lattice structure a diamond cell type is used, which is common in medical technology. For the later adaptation of the bone stiffness, the stiffness of the lattice structure with different strut diameters are determined. The calculation of the stiffness is done by numerical simulations using Finite Element Methods (FEM). The simulations are validated with tensile and compression tests. Finally, the automated design of the implants is carried out with an in-house generated tool to adjust the strut diameters based on the bone density from patient data.

Parts of this work have been funded by the German ministry of education and research (BMBF) under grant number 13GW0116.
\end{abstract}

\section{Introduction}

Lattice structures are used both in lightweight design and in medical technology to adjust the stiffness of a component locally. In addition, porous lattice structures in medical implants are used to 
adapt the osseointegrative behavior (Bandyopadhyay et al., 2009; Emmelmann et al., 2011). By using additive manufacturing processes, periodic lattice structures can be adapted to a specific application instead of using stochastic pore structures (e.g. metal foams). Metal lattice structures can be fabricated additively using Laser Powder Bed Fusion (LPBF). (Rehme, 2009)

Despite a relatively high effort through several iteration steps in design and fabrication process, since the real mechanical properties of additive manufactured lattice structures are largely unknown, these structures are used as a functional element for the adjustment of stiffness, however, only in components that are subsequently manufactured in larger quantities. The innovation consists in creating a significant simplification of the design of lattice structures for the adaptation of stiffness by adjusting diameter sizes of struts based on specified suitable boundary or operational conditions (e.g. parameterization based on bone density values) to individualize the lattice structures for patient-specific characteristics.

Bone density values of vertebral bodies can be determined by imaging methods of medical diagnostics. These bone density values correlate with the mechanical stiffness of the vertebral bodies (Krimphove, 2001). The aim of this work is to develop individualized lattice structures by local adaptation of the stiffness (modulus of elasticity) of vertebral implants based on the stiffness of the bone and then, manufacturing of the obtained monolithic implant using LPBF. With these adapted implants, the healing process can be improved as shown in (Pobloth et al., 2018), where it is demonstrated that a softer implant results in faster bone healing due to controlled stress shielding.

Parts of this work have been funded by the German ministry of education and research (BMBF) under grant number 13GW0116.
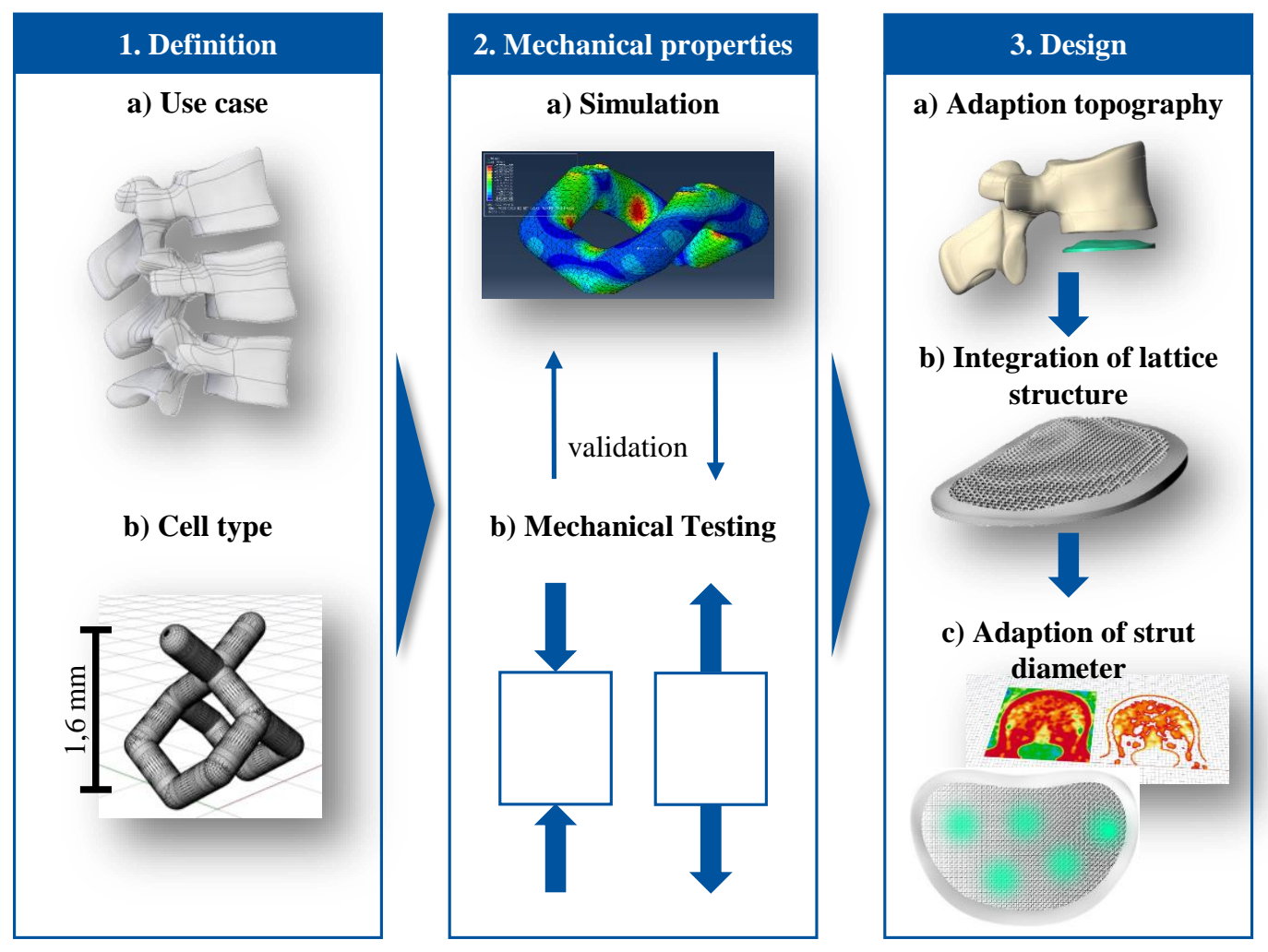

Figure 1: A schematic representation of the approach for creating individualized implants with adapted mechanical properties. 


\section{Approach}

A schematic representation of the approach is shown in Figure 1. For the demonstration of specific individualized implant, a vertebral body replacement implant is used (1a). A diamond cell type, commonly used in medical applications, is used as basis for the lattice structure generation (1b).

To create a lattice structure adapted to the patient data, the stiffness of the designed lattice structure needs to be determined. For this purpose, the diamond cell structure is examined using Finite Element Method (FEM) simulation to determine the modulus of elasticity as a function of cell parameters (2a). The FEM simulation model is validated using mechanical tests. For this purpose, tensile and compression tests are performed on lattice structures manufactured using LPBF and the results are compared with the stiffness values from the simulation $(2 \mathrm{~b})$.

To adapt the lattice structure to the bone stiffness individually, the designed geometry of the implant is first adapted to the topography of the patient's vertebral body (3a). Then, the diamond lattice structure is embedded into the implant (3b). In the final step, the lattice structure can be adapted to the stiffness derived from the bone density (3c).

\section{Results}

The stiffness of the diamond lattice structure is simulated and determined for different strut diameters. Strut diameters of $200 \mu \mathrm{m}, 320 \mu \mathrm{m}$ and $440 \mu \mathrm{m}$ are used. The simulation results show that the modulus of elasticity can be adjusted in a wide range $(0.83-5.64 \mathrm{GPa})$ by varying the strut diameter.

Tensile tests of the diamond lattice structures with a strut diameter of $320 \mu \mathrm{m}$ yield to a modulus of elasticity of $2.35 \mathrm{GPa}$. The modulus of elasticity of $2.87 \mathrm{GPa}$ calculated from the simulation deviates from the experimental value. This may be related to the fact that the simulation assumes ideal conditions (e.g. no surface roughness, homogeneous material properties, etc.). In compression tests a good match between simulation and experiment was achieved.
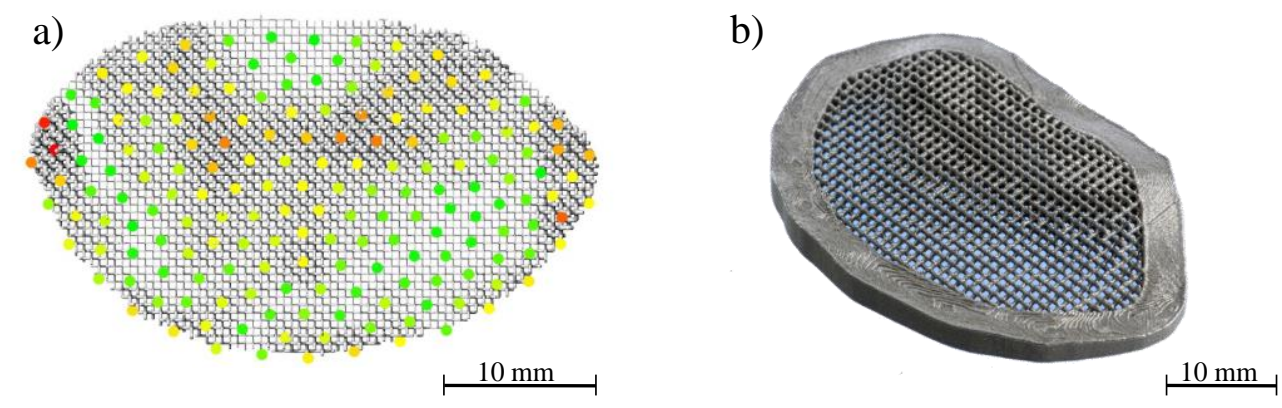

Figure 2: a) Top view representation of a lattice structure with adapted strut diameters with stiffness values represented by colored visualized points. b) Implant with lattice structures with adapted strut diameter manufactured using LPBF. 
Figure 2b) shows a lattice structure with adapted strut diameters including the imported point cloud in Figure 2a) with stiffness values of the bone density data of the patient. The strut diameter takes values from $50 \mu \mathrm{m}$ to $500 \mu \mathrm{m}$. To determine the strut diameter locally, the stiffness values in a circular area within the radius of $2 \mathrm{~mm}$ are used. The stiffness values are shown in color in Figure 2 in which points with low stiffness are shown in green and high stiffness values are visualized as red points. The design of these adapted lattice structures is automated using a point cloud derived from bone density with stiffness values.

\section{Discussion}

The paper represents a procedure to design lattice structures tailored for given stiffness values. These lattice structures are an inherent part of the final product (implant with embedded lattice structures) to improve the conventionally-manufactured implant (Pobloth et al., 2018). The simulation-based approach was chosen as it is faster and more economical than a purely experimental approach (Zargarian et al., 2016).

There are two variants for adapting a lattice structure to the topography of the vertebral body. On one hand, the lattice structure can be trimmed to the outer surface of the topography (non-conformal lattice structure). On the other hand, the lattice structure can be adapted so that they follow the topography and thus, the cell nodes are generated at the outer surfaces (conformal lattice structure). In addition to constant values within the lattice structure, the diameters of the struts can also have a gradient or can be adjusted locally. A challenge would be the prediction of mechanical properties of topologically adapted and gradient lattice structures, which requires further research.

\section{References}

Bandyopadhyay, A., Krishna, B. V., Xue, W. \& Bose, S. (2009). Application of laser engineered net shaping (LENS) to manufacture porous and functionally graded structures for load bearing implants. Journal of Materials Science: Materials in Medicine, 20 (1), 29.

Emmelmann, C., Scheinemann, P., Munsch, M. \& Seyda, V. (2011). Laser additive manufacturing of modified implant surfaces with osseointegrative characteristics. Physics Procedia, 12, 375-384.

Krimphove, M. (2001). Zum Zusammenhang zwischen mechanischer Belastbarkeit und Knochendichte von Lendenwirbelkörpern. Bochum.

Pobloth, A.-M., Checa, S., Razi, H., Petersen, A., Weaver, J. C., Schmidt-Bleek, K., Windolf, M., Tatai, A. Á., Roth, C. P. \& Schaser, K.-D. (2018). Mechanobiologically optimized 3D titaniummesh scaffolds enhance bone regeneration in critical segmental defects in sheep. Science translational medicine, 10 (423).

Rehme, O. (2009). Cellular design for laser freeform fabrication. Cuvillier Göttingen.

Zargarian, A., Esfahanian, M., Kadkhodapour, J. \& Ziaei-Rad, S. (2016). Numerical simulation of the fatigue behavior of additive manufactured titanium porous lattice structures. Materials Science and Engineering: C, 60, 339-347. 